\title{
SpecialNewsFeature
}

\section{BUILDING THE FUTURE FROM 3D}

\section{BLUEPRINTS}

\section{D printed biomaterials are increasingly used in cell cultures and drug screens. Given the ease of creating artificial tissues, will this technique revolutionize biomedicine and organ implants in the future?}

A bout a decade ago, Adam Engler, now a professor at the University of California San Diego, demonstrated that stem cells differentiated into different cell types depending on the mechanical properties of their substrate (1). Cells cultured on super soft gels mimicking brain tissue differentiated into neurons, while those cultured on gels of muscle-like intermediate stiffness differentiated into muscle cells; cells cultured on gels matching the stiffness of collagenous bone differentiated into osteoblasts.

Engler performed his foundational biomechanical experiments on cells cultured on 2D gels, but researchers soon tested the hypotheses in 3D. In 2016, Vishal Chaturvedi, a postdoctoral researcher at the University of Melbourne, Australia, cultured skeletal muscle cells on 3D scaffolds made of silk fibroins that differed in their mechanical properties from four different silkworm species. Although cells adhered to all of the scaffolds, he observed well-aligned myotubes, which are characteristic of muscle cells, only in the cells grown on silk scaffolds that matched the cellular elasticity (2). Thus, even in $3 \mathrm{D}$, "It is pertinent to match the elasticity of the scaffold with the cells that you are trying to proliferate or differentiate," said Chaturvedi.

As researchers continued their efforts to mimic natural structures, they found that it was difficult to apply conventional methods to construct scaffolds that fit all of their criteria: biocompatibility, defined stiffness, precise pore size, and homogeneous cell distribution. At the same time, 3D printing emerged as a promising technology. Printing with biomaterials offered flexibility in material choice and design for preparing intricate scaffolds from innovative materials, so researchers began to gravitate towards the technology.

\section{The Rise of 3D Printed Biomaterials}

Printing scaffolds for some cell types is simpler than for others. For instance, 3D printing has been successfully used for printing tumor models (3), skeletal scaffolds (4), and human scale tissue products (5), but creating soft scaffolds that match brain tissue while still being able to withstand the pressure of adherent cells has been particularly challenging. Daniele Dini, professor at Imperial College, London, was keen to overcome this challenge. He recently led a team to create super soft cryogenic hydrogels matching the softness of brain tissues (6).

The biggest challenge in creating super soft hydrogels is that the main component is water, so the structure needs to be frozen or given enough time to polymerize before it can be seeded, according to Dini. "The whole idea is that in order to create brain-like soft material, you need to allow interactions within polymers that are part of the solid structure," he said.

For their experiments, Dini's team used a composite hydrogel mixture as the ink and rapidly cooled it below its freezing point using dry ice. The researchers mechanically characterized the resulting high-quality $3 \mathrm{D}$ geometrical porous structures using compressibility tests to confirm a stiffness of $<1 \mathrm{kPa}$ (the stiffness of brain tissue), a novel achievement in $3 \mathrm{D}$ printed scaffolds. Next, the team seeded human skin cells 


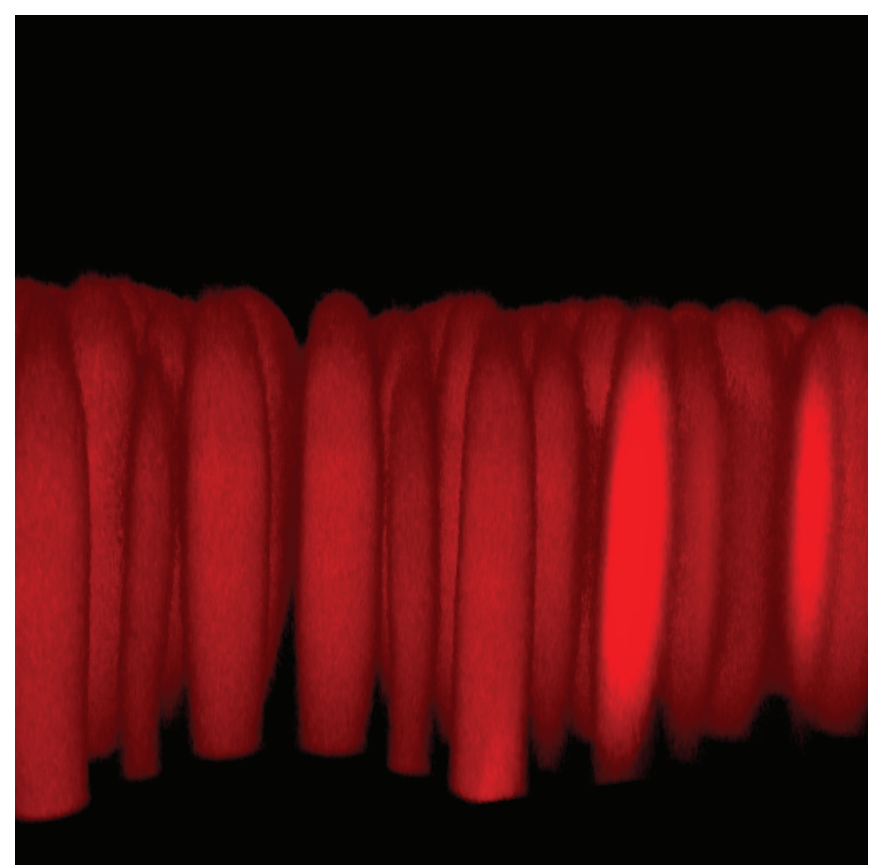

A three-dimensional rendering of a 3D-printed, vertically oriented artificial axon bundle from a confocal z-stack.

Credit: D. Espinosa-Hoyos, A. Jagielska, and K.J. Van Vliet, Massachusetts Institute of Technology.

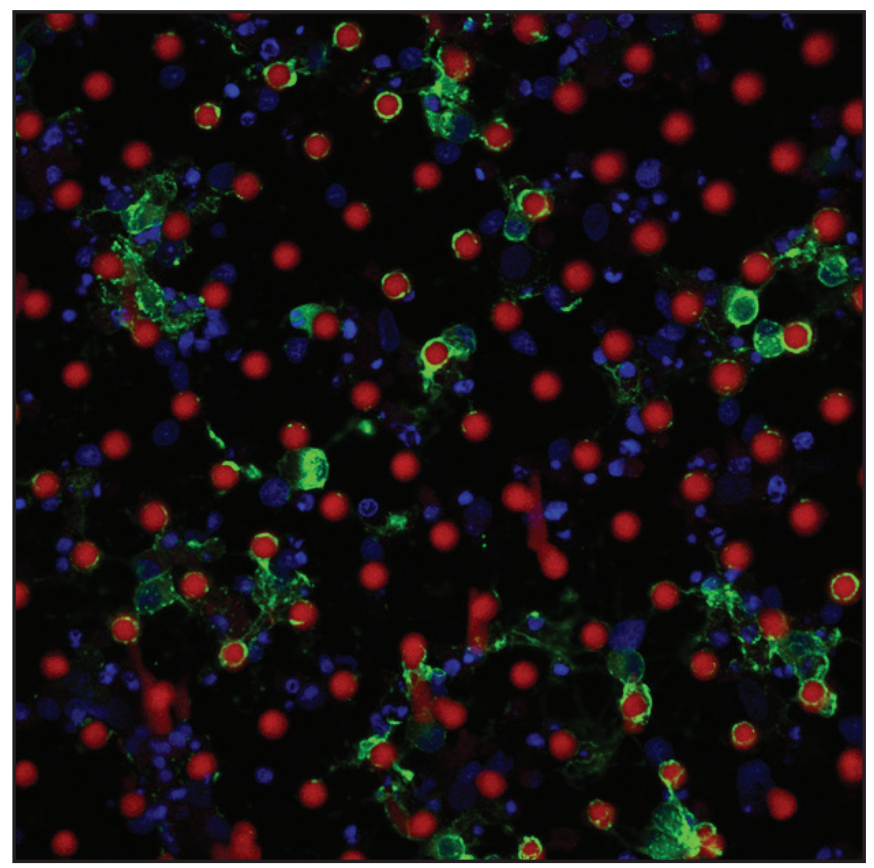

Mature oligodendrocytes wrap membranes rich in myelin basic protein (green rings) around artificial axons (red) that match key biochemical, physical, and mechanical properties of biological axons. Projection of a confocal z-stack. Credit: D. Espinosa-Hoyos, A. Jagielska, and K.J. Van Vliet, Massachusetts Institute of Technology.

on the scaffold and demonstrated its biocompatibility for future biomedical applications.

In the future, Dini plans to make larger 3D scaffolds for seeding different types of cells for prolonged culture. However, further research is required to show the full feasibility of this technique for creating real tissues before proceeding to animal studies, Dini cautioned.

\section{Beyond Scaffolds: Printing Artificial Cells}

Scaffolds are useful for mimicking the extracellular matrix, but in some cases, cell functionality relies on the architecture of other nearby cells. Krystyn Van Vliet, a professor at Massachusetts Institute of Technology, works on understanding how changes in environmental cues, such as stiffness, $\mathrm{pH}$, or structural arrangement following traumatic brain injury, affect the function of oligodendrocytes, the cells that form the myelin sheath around axons.

Van Vliet wanted to visualize myelin formation in vitro. " $2 \mathrm{D}$ gels could never get to a point where you could watch this process," she said. There is a big difference between testing whether cells can produce a membrane and testing whether this membrane can actually wrap around an axon.

Van Vliet realized that she and her team needed a 3D culture system complete with axons around which oligodendrocytes could grow. Axons cultured from brain tissues are not viable for long periods in vitro, so Van Vliet's team decided to engineer 3D printed artificial axons (7).

Designing a thin, self-holding, soft polymer that matched the stiffness of axons was quite a challenge. To achieve the correct geometry and mechanics, Daniela Espinosa-Hoyos, the first author of the study, tested different polymers to design a new polymer ink that yielded a fiber with a diameter of about one micron. The fiber had extremely low stiffness, but it remained freestanding so that oligodendrocytes cells could wrap myelin membranes around it.

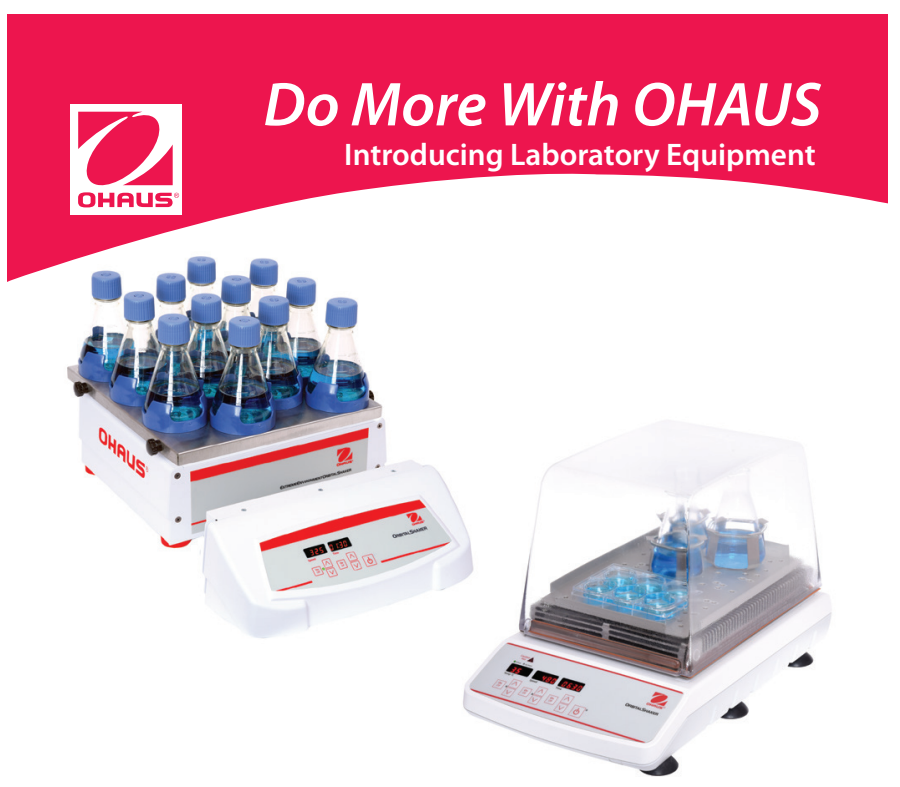

\section{Shake It Up With OHAUS}

From extreme environments requiring 100\% humidity to incubating models with accessories designed to handle a broad range of sample sizes, OHAUS has a wide variety of shakers engineered to meet every need in your cell culture workflow. Don't limit yourself to just measurement- Do More.

Visit www.ohaus.com to learn more 


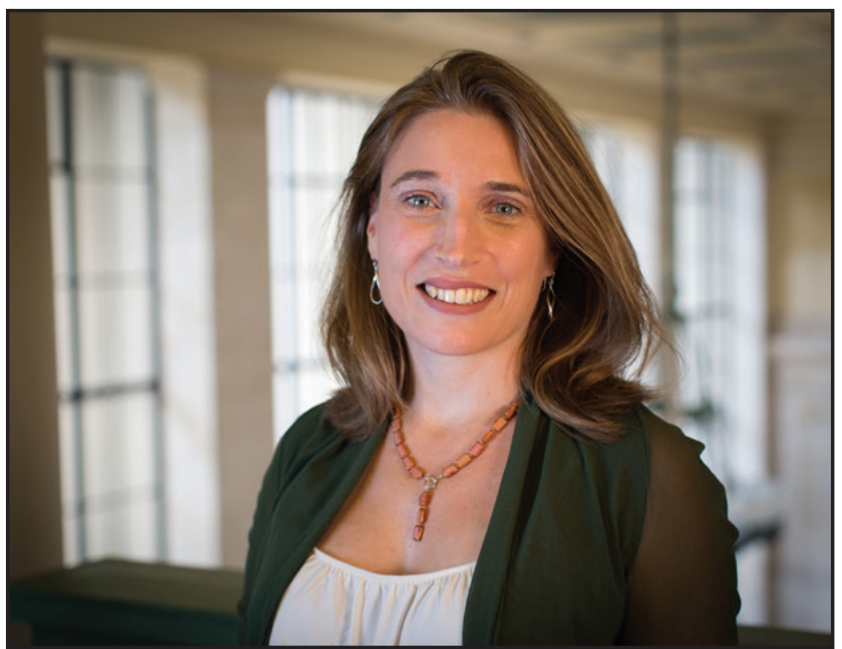

Krystyn Van Vliet, Massachusetts Institute of Technology.

The team plans to continue focusing on studying the myelination process using their in vitro model. According to Van Vliet, these axons make excellent tools for screening drugs that promote rewrapping of oligodendrites. "You want to ask this question not on a flat plastic dish, but in a 3D environment," she said.

\section{Projecting the Future in 3D}

The benefits and technological developments in new 3D cultures imply that 2D cultures might soon be obsolete, but experts believe otherwise. The expense of 3D technology, difficulties with reproducibility, and a lack of automation still hinder researchers from adopting 3D culture as the primary method for cellular studies. However, many researchers believe that commercial intervention will solve these major technical hurdles within the next 2-5 years.

"We are seeing a greater interest in 3D cell culture and a growth in demand for related technologies," said Klaus Bischoff, head of research solutions at MilliporeSigma. "However, the scientific community has spent the last 30-40 years building data with 2D cell culture models. It will take some time to begin to amass this level of knowledge for 3D models."

Bischoff believes that the need for better tumor models, vascularized tissues, and studies of absorption, distribution, metabolism, excretion, and toxicity will fuel future enhancements in scalability, reproducibility, and 3D model standardization.

While drug screening remains the top application for 3D cultures, 3D printed scaffolds show promise for tissue engineering, especially when using a patient's own cells to minimize the chances of rejection. Thus, 3D printed organs may not be far-off for patients suffering from organ dysfunction. In fact, companies such as Organovo and Prellis Biologics are already printing functional human tissues using 3D bioprinting. The latter aims to print fully functional organs in the next 4-6 years, and hopes to start clinical trials of their 3D printed islet cells for diabetic patients by 2021.

The freedom of choosing the design and material of the ink is the strength of this method, according to Van Vliet. "We don't use off-the-shelf materials; we make materials for the shelf. The more variety [of materials] the community has, the bigger that shelf is, and the more the applications will extend beyond what we can conceive of today," she said.

\section{References}

1. Engler, A. J. et al. 2006. Matrix elasticity directs stem cell lineage specification. Cell 126(4): 677-689.

2. Chaturvedi, V. et al. 2017. Silk fibroin scaffolds with muscle-like elasticity support in vitro differentiation of human skeletal muscle cells. Journal of tissue engineering and regenerative medicine 11.11: 3178-3192.

3. Zhao Y., Yao R., Ouyang L., Ding H., Zhang T., Zhang K., Cheng S., Sun W. 2014. Three-dimensional printing of Hela cells for cervical tumor model in vitro. Biofabrication. 6(3):035001.

4. www.biotechniques.com/news/365732

5. Kang H.W., Lee S.J., Ko I.K., Kengla C., Yoo J.J., Atala A. 2016. A 3D bioprinting system to produce human-scale tissue constructs with structural integrity. Nat Biotechnol.

6. Tan, Z. et al. 2017. Cryogenic 3D Printing of Super Soft Hydrogels. Scientific reports 7.1:16293.

7. Espinosa-Hoyos, D. et al. 201. Engineered 3D-printed artificial axons. Scientific reports 8.1:478.

Written by Meenakshi Prabhune, PhD

BioTechniques 64:96-99 (March 2018) doi 10.4155/btn-2017-0113

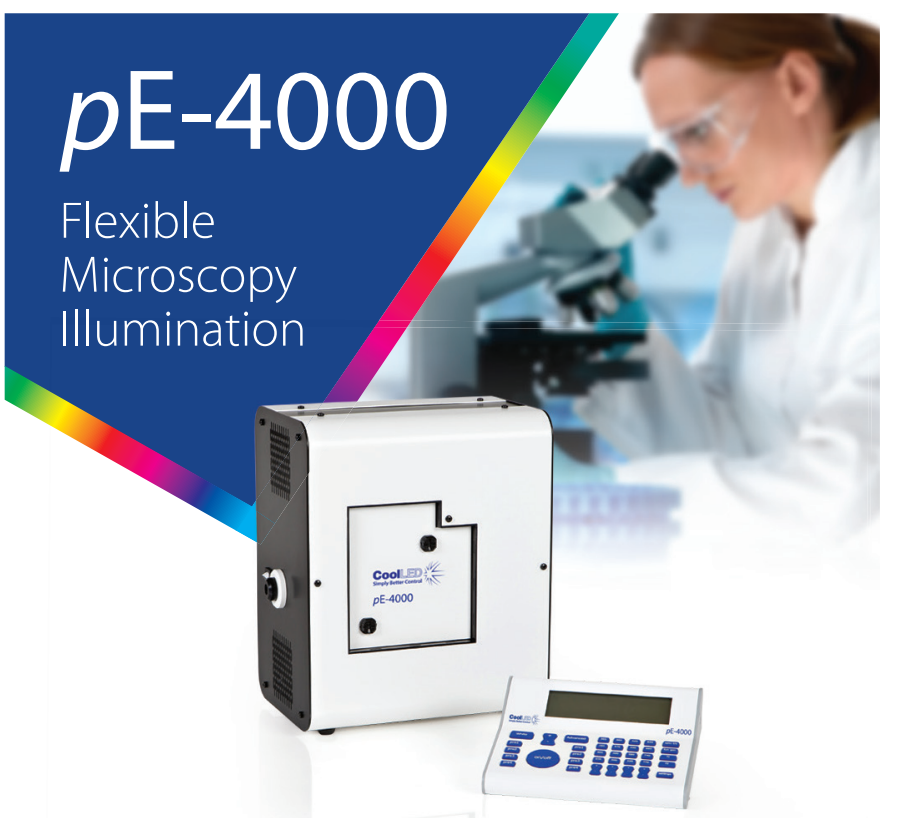

Broadest Fluorophore Coverage -

DAPI-Cy7 and beyond...

Enhanced Intensity

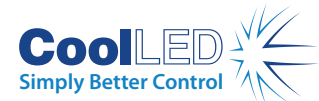

+44 (0)1264323040 (Worldwide) 1-800-877-0128 (USA/Canada) e: info@CoolLED.com 